\title{
Accelerated Phase-Cycled SSFP Imaging With Compressed Sensing
}

\author{
Tolga Çukur, Member, IEEE
}

\begin{abstract}
Balanced steady-state free precession (SSFP) imaging suffers from irrecoverable signal losses, known as banding artifacts, in regions of large $B 0$ field inhomogeneity. A common solution is to acquire multiple phase-cycled images each with a different frequency sensitivity, such that the location of banding artifacts are shifted in space. These images are then combined to alleviate signal loss across the entire field-of-view. Although high levels of artifact suppression are viable using a large number of images, this is a time costly process that limits clinical utility. Here, we propose to accelerate individual acquisitions such that the overall scan time is equal to that of a single SSFP acquisition. Aliasing artifacts and noise are minimized by using a variable-density random sampling pattern in k-space, and by generating disjoint sampling patterns for separate acquisitions. A sparsity-enforcing method is then used for image reconstruction. Demonstrations on realistic brain phantom images, and in vivo brain and knee images are provided. In all cases, the proposed technique enables robust SSFP imaging in the presence of field inhomogeneities without prolonging scan times.
\end{abstract}

Index Terms-Banding artifact, compressed sensing, magnetic resonance imaging (MRI), phase cycling, random undersampling, steady-state free precession (SSFP), variable density.

\section{INTRODUCTION}

B ALANCED steady-state free precession (SSFP) sequences [1] are utilized for fast imaging in a broad range of magnetic resonance imaging (MRI) applications including cardiac imaging [2]-[4], angiography [5], [6], musculoskeletal imaging [7], [8], interventional imaging [9], [10], cell tracking [11], [12], and functional imaging [13], [14]. Compared to gradient or spin-echo sequences, the utility of SSFP in rapid imaging arises from its relatively short repetition times (TR), and high signal-to-noise ratios (SNRs) per unit scan time [15]. However, these advantages come at the cost of an MRI signal whose magnitude is strongly perturbed by magnetic field inhomogeneities across the imaging sample. In SSFP images, field inhomogeneities routinely encountered in practice can cause characteristic signal voids, known as banding artifacts. Thus, SSFP sequences suffer from irrecoverable loss of image information in regions with large off-resonant frequencies [16].

Manuscript received June 20, 2014; revised July 31, 2014; accepted August 06, 2014. Date of publication August 12, 2014; date of current version December 24,2014 . This work was supported by in part by a TUBITAK 2232 Fellowship (113C011), and in part by a Marie Curie Actions Career Integration Grant (PCIG13-GA-2013-618101). Asterisk indicates corresponding author.

The author is with the Department of Electrical and Electronics Engineering and the National Magnetic Resonance Research Center, Bilkent University, Bilkent, 06800 Ankara, Turkey (e-mail: cukur@ee.bilkent.edu.tr).

Color versions of one or more of the figures in this paper are available online at http://ieeexplore.ieee.org.

Digital Object Identifier 10.1109/TMI.2014.2346814
A number of successful strategies have been proposed to alleviate banding artifacts [17]-[26], but multiple-acquisition SSFP imaging is by far the most commonly used method [17]. The multiple-acquisition method collects multiple SSFP images across which banding artifacts appear in nonoverlapping locations. The location of banding artifacts are altered by shifting the frequency response of SSFP acquisitions. This shift can be implemented either by changing the center frequency directly, or by altering the radio-frequency (RF) phase-increments between successive TRs. These methods are generally referred to as phase cycling. Individual SSFP images are then combined using an algorithm that retains tissue signals while reducing banding artifacts [18]-[21], [27]. It is desirable to maximize the number of SSFP acquisitions in order to increase the level of artifact suppression. Unfortunately, this also diminishes the speed advantage of SSFP sequences and increases sensitivity to motion artifacts.

Here, we propose a new technique that effectively reduces banding artifacts by combining a relatively large number of phase-cycled acquisitions. These acquisitions are randomly undersampled in order to maintain a total scan time equal to that of a single SSFP acquisition. To minimize aliasing energy and maximize image resolution, we propose to employ disjoint sampling patterns across multiple acquisitions as opposed to a common pattern. Finally, the undersampled data are reconstructed using compressed sensing [28]-[30], and later combined using the p-norm method to achieve near-optimal banding-artifact reduction and SNR [21].

\section{METHODS}

To maintain a fixed scan time regardless of the total number of phase-cycled acquisitions $(N)$, individual acquisitions must be accelerated by a factor of $N$. Any reconstruction of such undersampled data will contain residual aliasing and noise. We reasoned that if a common sampling pattern is used for all acquisitions, then these residual artifacts will add up coherently. In contrast, if disjoint sampling patterns that contain partly distinct subsets of k-space samples are used, then residual artifacts will interfere destructively. Thus, we hypothesized that employing distinct patterns across separate acquisitions will improve image quality compared to a common pattern. We evaluted this hypothesis for both uniform-density and variable-density sampling patterns. A nonlinear reconstruction was employed to recover image structure from undersampled data using appropriate sparsifying transformations. Finally, the reconstructed SSFP images were combined to reduce banding artifacts. Maximum-intensity, sum-of-squares, and p-norm 
combination methods were tested. The following sections describe in detail the procedures used to demonstrate the proposed technique.

\section{A. Multiple-Acquisition SSFP Imaging}

The multiple-acquisition method collects a total of $N$ SSFP images, each with a different phase increment $\Delta \phi_{n}$, where $n \in$ [1 N]. The MRI signal acquired using each phase-increment is given as [31]

$$
M_{n}(r)=M_{o}(r) \frac{e^{i\left(\phi(r)+\Delta \phi_{n}\right) \frac{T E}{T R}} \cdot\left(1-A(r) e^{-i\left(\phi(r)+\Delta \phi_{n}\right)}\right)}{1-B(r) \cos \left(\phi(r)+\Delta \phi_{n}\right)}
$$

where TE is the echo time, TR is the repetition time, $r$ is the spatial location, and $\phi(r)$ is the spatial distribution of phase accrual over a single TR due to B0 field inhomogeneity. Meanwhile, $M_{o}(r), A(r)$ and $B(r)$ are terms that depend on several imaging and tissue relaxation parameters (see Appendix for details), but do not depend on $\phi(r)$ or $\phi_{n}$.

The goal of the multiple-acquisition method is to retain SSFP tissue contrast while removing the off-resonant frequency dependence of the signal in (1). In other words, the resulting image should be equivalent to a fully-refocused SSFP acquisition where $\phi(r)+\Delta \phi_{n}=\pi$ at all locations across the image

$$
\left|M_{\text {desired }}(r)\right|=\left|M_{o}(r)\right| \cdot\left|\frac{1+A(r)}{1+B(r)}\right| .
$$

In principle, if a large number of images are collected with $\Delta \phi_{n}$ densely sampling the range $\left[\begin{array}{ll}0 & 2 \pi\end{array}\right]$, the off-resonant frequency shift for any given voxel will be refocused in at least one phasecycled image. However, the phase-cycled image (equivalently $\Delta \phi_{n}$ ) that eliminates signal loss at a given voxel cannot be determined a priori unless the value of $\phi(r)$ at that voxel is known.

Instead, the multiple-acquisition method relies on the observation that the signal intensity should be higher for refocused SSFP signals than for signal voids. Therefore, banding artifacts can be reduced by a weighted combination of multiple phase-cycled images. Several combination methods have been proposed, including maximum-intensity (MI) [18], sum-of-squares (SOS) [19], and p-norm [22] combinations

$$
\begin{aligned}
& M_{\text {combined }}(r) \\
& =\left\{\begin{array}{ll}
\max _{n}\left|M_{n}(r)\right|, & \text { maximum-intensity } \\
\left(\sum_{n}\left|M_{n}(r)\right|^{2}\right)^{\frac{1}{2}}, & \text { sum-of-squares } \\
\left(\sum_{n}\left|M_{n}(r)\right|^{p}\right)^{\frac{1}{p}}, & \mathrm{p}-\text { norm }
\end{array} .\right.
\end{aligned}
$$

MI is the most effective among the techniques considered here in reducing banding artifacts, but each voxel intensity in the combined image is determined solely by intensity in one phasecycled image. Thus, MI is suboptimal in terms of SNR efficiency. SOS yields near-optimal SNR but it is less effective in reducing banding artifacts. Meanwhile, the p-norm method converges to MI as $p$ approaches infinity (i.e., better artifact reduction for large $p$ ), and it converges to SOS as $p$ approaches 2 (i.e., higher SNR efficiency). Previous work has indicated that $p \approx 4-5$ maintains a favorable trade-off between artifact suppression and SNR efficiency [21], [22].

\section{B. Sampling Patterns}

To maintain an overall scan time equal to the duration of a single SSFP acquisition, each phase-cycled acquisition was $\mathrm{N}$-fold accelerated through undersampling. Both uniform-density and variable-density sampling patterns were generated across k-space. Two separate sampling strategies were then implemented across phase-cycles: a single pattern for all acquisitions, or alternatively a unique pattern for each acquisition.

1) Uniform-Density Sampling: Two-dimensional uniformdensity sampling patterns were generated. Because the energy in MR images is concentrated at low-spatial frequencies, the central $10 \%$ of k-space was fully sampled. To undersample the remaining portions of k-space, a center-out square-spiral trajectory was first generated that spans through the entire k-space [32]. An acceleration factor of $N$ was then achieved by sampling every $N$ th point along this trajectory. Note that the spiral trajectory is not used to specify the order of data collection, but rather to generate a pseudo-random sampling pattern on a Cartesian grid. This sampling strategy yields trajectories with similar shapes to Caipirinha trajectories [33], and thus reduces aliasing energy while maintaining near-isotropic sampling density in two dimensions.

2) Variable-Density Sampling: Variable-density sampling patterns were also generated in two dimensions, with the central $10 \%$ of k-space fully sampled. A sampling density was first constructed that gradually diminishes towards the periphery of $\mathrm{k}$-space based on a polynomial [29], [30], [34]

$$
P\left(k_{r}\right)= \begin{cases}a_{1}\left(1-k_{r}\right)^{d}+a_{2}, & \text { for } k_{r}>0.1 \\ 1, & \text { for } k_{r}<0.1\end{cases}
$$

where $k_{r}$ is the $\mathrm{k}$-space radius (normalized to a maximum of $1), d$ is the degree of the polynomial, and $a_{1,2}$ are constants selected to yield a desired acceleration factor (R). For a given R, separate sampling densities were constructed using polynomial degrees varying from 1 to 8 . For each $d, a_{1,2}$ that maintain the target acceleration were identified using a binary search algorithm. A probability density function (PDF) was then created by normalizing the sampling density to a sum of 1 over the entire k-space. The resulting PDF was used to generate random sampling patterns.

A total of 2000 random patterns were generated ( 250 patterns for each $d$ ) using a Monte Carlo procedure. The point spread function (PSF) for each pattern was simulated assuming an impulse object located at the center of the image. Undersampled k-space data were zero-filled and inverse Fourier transformed to compute the PSF. Aliasing energy was computed as the magnitude sum of all noncentral pixels in the PSF. The optimal pattern among 2000 candidates was selected to yield minimal aliasing energy. Repeated Monte Carlo procedures indicated that 2000 iterations were sufficient to converge on the same aliasing-energy minimum.

3) Common Versus Disjoint Sampling: In the common sampling strategy, a single uniform or variable-density sampling pattern was generated. This specific pattern was used to under- 
TABLE I

DESIGN PARAMETERS OF SAMPLING PATTERNS

\begin{tabular}{c|ccc|cccc|cc} 
& \multicolumn{4}{|c|}{ Phantom Data } & \multicolumn{3}{c}{ Brain Data } & \multicolumn{2}{c}{ Knee Data } \\
\hline Number of Acq. & $d$ & $a_{1,2}$ & Corr. & $d$ & $a_{1,2}$ & Corr. & $d$ & $a_{1,2}$ \\
\hline \hline $\mathbf{N}=\mathbf{2}$ & 8 & $1.23,0.47$ & $0.035 \pm 0.000$ & 6 & $1.03,0.45$ & $0.042 \pm 0.000$ & 6 & $1.03,0.45$ & $0.040 \pm 0.000$ \\
\hline $\mathbf{N}=\mathbf{4}$ & 8 & $1.84,0.21$ & $0.069 \pm 0.001$ & 6 & $1.52,0.19$ & $0.088 \pm 0.002$ & 6 & $1.52,0.19$ & $0.074 \pm 0.004$ \\
\hline $\mathbf{N}=\mathbf{6}$ & 8 & $2.02,0.13$ & $0.108 \pm 0.002$ & 6 & $1.67,0.11$ & $0.135 \pm 0.004$ & 6 & $1.67,0.11$ & $0.105 \pm 0.004$ \\
\hline $\mathbf{N}=\mathbf{8}$ & 8 & $2.16,0.07$ & $0.148 \pm 0.003$ & 6 & $1.75,0.07$ & $0.184 \pm 0.005$ & 6 & $1.75,0.07$ & $0.137 \pm 0.004$ \\
\hline
\end{tabular}

Design parameters of sampling patterns generated for phantom, brain and knee data. The polynomial order of the density function $(d)$, density coefficients $\left(a_{1,2}\right)$, and the correlation (mean \pm s.d. across $\mathrm{N}$ patterns) between distinct patterns for disjoint sampling are listed.

sample all of the $N$ phase-cycled SSFP acquisitions. However, with disjoint sampling, a distinct sampling pattern was generated for each acquisition. For uniform-density patterns, the square-spiral trajectory was interleaved into $N$ nonoverlapping segments, and each segment was taken as a distinct pattern. For instance, the first pattern contained samples $\{1, N+1, \ldots\}$, and the second pattern contained samples $\{2, N+2, \ldots\}$. For variable-density patterns, $N$ separate patterns were selected using a slightly modified Monte Carlo procedure. The first pattern was selected among 2000 candidates to yield minimal aliasing energy as in the original procedure. To select the second pattern, we first identified candidates in the fifth percentile according to aliasing energy. We then calculated Pearson's correlation between the first pattern and the fifth percentile of candidates for the second pattern. Because sampling patterns are in matrix form, each matrix was converted into a column vector prior to correlation measurements. We selected the candidate yielding the lowest correlation (i.e., least amount of similarity) with the first pattern. In general, the nth pattern was selected as the candidate that yields the lowest average correlation with the $(n-1)$ previously selected patterns. This procedure is similar to the use of correlation to measure the coherence of system matrices in linear algebra [35]. The design parameters of the sampling patterns for phantom and in vivo experiments reported in this work are listed in Table I.

\section{Compressed-Sensing Reconstructions}

Due to the inherent sparsity of MRI images, compressedsensing reconstructions can recover images from heavily undersampled MRI datasets [29]. This recovery relies on several critical conditions. First, images must be accurately represented by a relatively small number of coefficients in a linear transform domain. The appropriate transform can be a wavelet or identity transformation based on the underlying image structure [29]. Second, undersampling should produce incoherent aliasing artifacts in this transform domain, with minimal resemblance to the actual image structure. Lastly, a nonlinear algorithm should be used to simultaneously enforce the compressibility of the reconstructed images and the consistency of the reconstructions with the acquired data.

In this work, compressed-sensing reconstructions were implemented through the following optimization problem [29], [30], [34]:

$$
\underset{m}{\operatorname{minimize}}\left\|\left(F_{u} m-Y\right)\right\|_{2}+\lambda_{1}\|\Phi(m)\|_{1}+\lambda_{2} T V(m) .
$$

Here, $m$ denotes the reconstructed image. The first term enforces data consistency by penalizing the $\ell^{2}$-norm difference between the reconstruction $\left(F_{u} m\right)$ and the acquired data $(Y)$ in $\mathrm{k}$-space. The second term enforces compressibility by penalizing the $\ell^{1}$-norm of the image in the transform domain, $\Phi(m)$. The third term reduces aliasing artifacts and noise in the reconstructed image by penalizing the sum of spatial finite differences of the image, $T V(m)$. Relatively small penalty weights, $\lambda_{1,2}$, were utilized to minimize undesired loss of image features with low contrast and/or small size. Separate reconstructions were performed while $\lambda_{1,2}$ were independently varied from 0 to $2 \times 10^{-2}$ in steps of $5 \times 10^{-4}$. The optimal penalty weights were selected to achieve the highest image quality, measured in terms of peak signal-to-noise ratio and structural similarity that are explained in Section II-D. The optimization was solved using an iterative conjugate-gradient method, and the iterations were stopped when the improvement in the objective fell below $0.1 \%$ [29]. Reconstruction times for simulated phantom and in vivo images reported here are listed in Table IV.

\section{Assessment of Image Quality}

1) Peak SNR and Structural Similarity: The quality of the reconstructed images was assessed by calculating the meansquared error (MSE) between reconstructed images and a reference. The maximum signal intensity in the reference image was normalized by the MSE to determine the peak signal-to-noise ratio [36]

$$
\text { PSNR }=10 \log _{10}\left(\frac{\max _{r}\left|m_{\mathrm{ref}}(r)\right|^{2}}{\frac{1}{l} \sum_{r=r_{1}}^{r_{l}}\left|m(r)-m_{\mathrm{ref}}(r)\right|^{2}}\right)
$$

where $m_{\text {ref }}$ denotes the reference image, $r_{1 . . l}$ denote pixel locations, and $l$ is the number of pixels. Higher PSNR values indicate a closer match between the reference and reconstructed images. Note that PSNR is a summary statistic for the quality of the entire image, as opposed to conventional SNR measurements over a specific region-of-interest. To estimate the reconstruction errors perceived by the human visual system, the mean structural similarity index (SSIM) was calculated between the reconstructed and reference images

$$
\operatorname{SSIM}=\frac{1}{X Y} \sum_{x=1}^{X} \sum_{y=1}^{Y} \frac{\left(2 \mu_{x} \mu_{y}+c_{1}\right)\left(2 \sigma_{x y}+c_{2}\right)}{\left(\mu_{x}^{2}+\mu_{y}^{2}+c_{1}\right)\left(\sigma_{x}^{2}+\sigma_{y}^{2}+c_{2}\right)} .
$$

Here, $x$ is a $20 \times 20$ image window from the reconstructed image ( $X$ being the total number of windows), $y$ is a $20 \times 20$ image window from the reference image ( $Y$ being the total 
number of windows), $\mu$ is the average pixel intensity in an image window, $\sigma$ is the covariance of pixel intensities across image windows. SSIM was computed using stabilization parameters of $c_{1}=10^{-4}$ and $c_{2}=9 \times 10^{-4}$, and a Gaussian smoothing kernel of 10-pixel width and 5-pixel standard deviation [37]. Higher SSIM values indicate a closer perceptual match between the reference and reconstructed images. Note that arbitrary scalings of image contrast and brightness can lead to inadvertent deviations in PSNR and SSIM measurements. Thus, histograms of the reconstructed and reference images were matched prior to image quality assessments. For this purpose, equispaced histograms of reference and reconstructed images (both normalized to a maximum intensity of 1) were separately calculated. An intensity transformation was then computed for the reconstructed image that matches the histogram counts between the reference and reconstructed images.

2) Spatial Resolution: To assess the effect of sampling patterns on image resolution, the line spread function (LSF) of each sampling method was simulated separately. For this purpose, the modulation transfer function (MTF) was first measured using the slanted-edge method [38]. Images of a slantededge separating uniform high- (1) and low-intensity (0) regions were generated for edge orientations ranging from $0^{\circ}$ to $360^{\circ}$. Images were Fourier transformed, and undersampled using uniform- and variable-density patterns, and common and disjoint sampling across $N$ phase-cycled acquisitions.

For common sampling, each slanted-edge image was undersampled with a single common pattern. For disjoint sampling, each slanted-edge image was undersampled $N$ times with distinct patterns. To compute the MTF, undersampled data were density compensated in k-space, inverse Fourier transformed, and combined with the $\mathrm{p}$-norm method $(p=4)$. The spatial derivative of the MTF in the horizontal direction was calculated to derive the LSF. The total energy under the LSF was computed for each separate sampling method, and for a fully-sampled acquisition as a reference [39]. Spatial resolution at each orientation was quantified by normalizing the LSF energy by that of the reference.

\section{E. Simulations}

To evaluate the proposed technique, a realistic digital brain phantom was used (http://www.bic.mni.mcgill.ca/brainweb). This phantom is constructed from high-resolution $\mathrm{T}_{1-}, \mathrm{T}_{2-}$, and proton-density weighted images, and it has a spatial resolution of $0.5 \times 0.5 \times 0.5-\mathrm{mm}^{3}$ [40]. The phantom provides the spatial distribution of each tissue across the brain volume, and the relative proportion of the tissues within each voxel.

To simulate brain images, phase-cycled SSFP signals for each tissue were first calculated according to (1). The following three-dimensional (3D) acquisition parameters were assumed: a flip angle of $\alpha=30^{\circ}, 5.0-\mathrm{ms} \mathrm{TR}, 2.5-\mathrm{ms} \mathrm{TE}$, and 12 phase-cycled acquisitions with the following increments $\Delta \phi=$ $(0, \pi / 4, \pi / 3, \pi / 2,2 \pi / 3,3 \pi / 4, \pi, 5 \pi / 4,4 \pi / 3,3 \pi / 2,5 \pi / 3,7 \pi / 4)$. A field-inhomogeneity map provided with the phantom was used to simulate off-resonant frequency shifts at each voxel. The mean off-resonant frequency across the brain volume was $0 \mathrm{~Hz}$, with a standard deviation of $62 \mathrm{~Hz}$. The following relaxation parameters were used: $\mathrm{T}_{1} / \mathrm{T}_{2}=1300 / 110 \mathrm{~ms}$ for
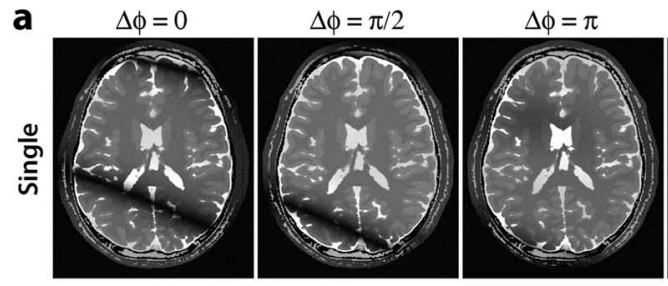

$\Delta \phi=3 \pi / 2$
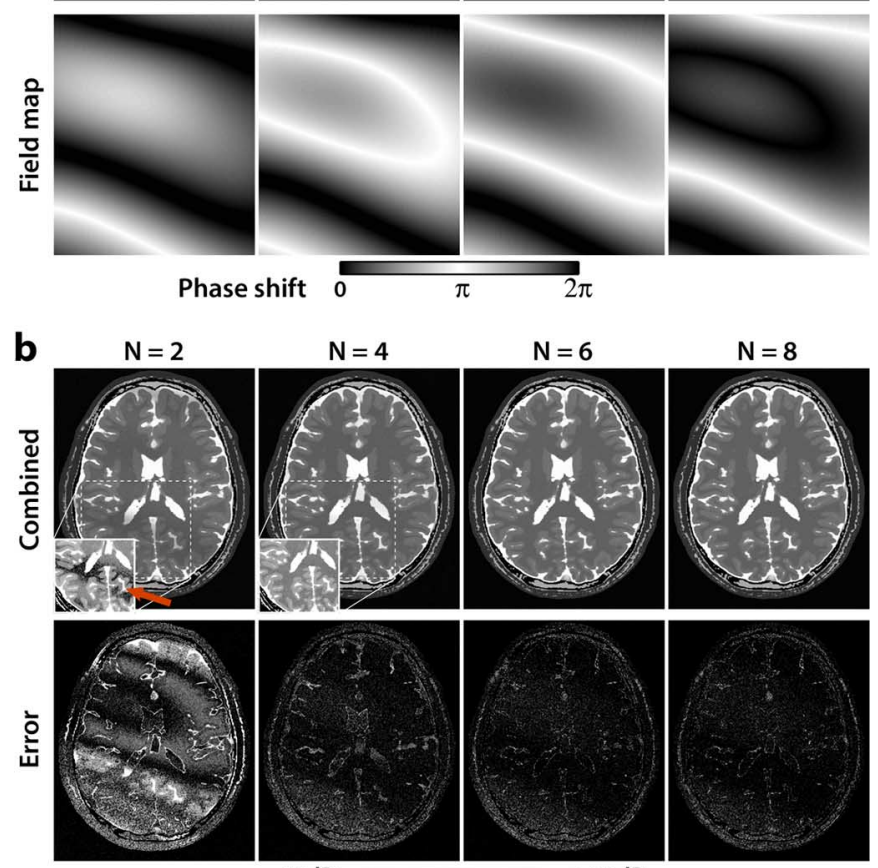

Fig. 1. Simulated SSFP images of the brain for $\alpha=30^{\circ}, 5.0-\mathrm{ms}$ TR 2.5-ms TE, and an off-resonant frequency distribution (mean: $0 \mathrm{~Hz}$, s.d. $62 \mathrm{~Hz}$ ). (a) SSFP images at different phase-cycling increments (top row), $\Delta \phi=(0, \pi / 2, \pi, 3 \pi / 2)$. The corresponding field maps that incorporate both B0 inhomogeneity and RF phase cycling display the phase shift induced over one TR (bottom row). Regions of zero phase-shift (modulo $2 \pi$ ) appear as signal voids in SSFP images, whereas pass-band SSFP signals are observed in regions of $\pi$ phase-shift (see color bar). (b) Total of $N$ separate phase-cycled images were combined to alleviate banding artifacts (top row). Display windowing within the white boxes has been adjusted to emphasize banding artifacts. Squared error between the combined images and a banding-free reference image are also shown (bottom row, see color bar for logarithmic scale). While residual banding artifacts are clearly visible for $N=2$ (red arrow), artifact suppression improves significantly for larger $N$.

gray matter, $\mathrm{T}_{1} / \mathrm{T}_{2}=1000 / 80 \mathrm{~ms}$ for white matter, $\mathrm{T}_{1} / \mathrm{T}_{2}=1200 / 250 \mathrm{~ms}$ for blood, $\mathrm{T}_{1} / \mathrm{T}_{2}=3000 / 1000 \mathrm{~ms}$ for cerebro-spinal fluid (CSF), $\mathrm{T}_{1} / \mathrm{T}_{2}=1400 / 30 \mathrm{~ms}$ for muscle, and $\mathrm{T}_{1} / \mathrm{T}_{2}=370 / 130 \mathrm{~ms}$ for fat [41], [42]. The complex SSFP signals for separate tissues were linearly summed, and complex white Gaussian noise was added to the images to simulate varying levels of image SNR (see Fig. 1(a) for representative images).

The simulated images were undersampled by a factor of $N$ using either uniform- or variable-density patterns in two phase-encode dimensions. To examine the effects of different anatomical views, three separate phase-encoding schemes were considered: anterior/posterior and right/left, superior/inferior and anterior/posterior, and superior/inferior and right/left. For common sampling, one specific pattern was used for all phase-cycled images. For disjoint sampling, a different sampling pattern was used for each image. The 
following acquisitions were used to reconstruct the brain images: $\Delta \phi=(0, \pi)$ for $N=2, \Delta \phi=(0, \pi / 2, \pi, 3 \pi / 2)$ for $N=4, \Delta \phi=(0, \pi / 3,2 \pi / 3, \pi, 4 \pi / 3,5 \pi / 3)$ for $N=6$, and $\Delta \phi=(0, \pi / 4, \pi / 2,3 \pi / 4, \pi, 5 \pi / 4,3 \pi / 2,7 \pi / 4)$ for $N=8$.

To prevent image blurring, undersampled data were compensated to account for changes in sampling density across k-space. For uniform-density patterns, data outside the central k-space was multiplied by the undersampling factor. For variable-density patterns, data were multiplied by the inverse of the probability density functions used for generating the sampling masks. The density-compensated data were then reconstructed using compressed sensing. An orthogonal wavelet transform -Daubechies 4- [43], $\lambda_{1}=0.003, \lambda_{2}=0.006$, and 60 iterations were observed to yield high-quality reconstructions. The CS reconstructions were finally combined using three methods: maximum-intensity, sum-of-squares, or p-norm combinations $(p=4)$.

Note that the simulations outlined above incorporate natural brain anatomy, B0 field inhomogeneity, noise, partial volume effects, and sampling artifacts. We also examined the effects of TR/TE, flip angle, and relaxation parameters. For this purpose, simulations were repeated for all possible combinations of the following set of parameters: $\mathrm{TR} / \mathrm{TE}=(5.0 / 2.5 \mathrm{~ms}, 10.0 / 5.0 \mathrm{~ms}, 15.0 / 7.5 \mathrm{~ms})$, $\alpha=\left(30^{\circ}, 60^{\circ}, 90^{\circ}\right)$, and $(-20 \%, 0 \%, 20 \%)$ deviation in $\mathrm{T}_{1} / \mathrm{T}_{2}$ ratios for all tissues. The aforementioned sampling and reconstruction strategies were employed in each case.

\section{F. In Vivo Experiments}

In vivo SSFP images of the brain were acquired on a $3 T$ Siemens Magnetom scanner (maximum gradient strength of 45 $\mathrm{mT} / \mathrm{m}$ and slew rate of $200 \mathrm{~T} / \mathrm{m} / \mathrm{s}$ ) using a 32-channel head coil. Images were acquired with a $3 \mathrm{D}$ balanced SSFP sequence. The imaging parameters were as follows: $218 \times 218 \times 218-\mathrm{mm}^{3}$ field-of-view, $0.85 \times 0.85 \times 0.85-\mathrm{mm}^{3}$ spatial resolution, $\alpha=30^{\circ}, 5.1-\mathrm{ms}$ TR, $2.65-\mathrm{ms} \mathrm{TE}$, left/right and anterior/posterior phase-encode dimensions. Eight phase-cycled images were acquired with $\Delta \phi=(0, \pi / 4 \pi / 2,3 \pi / 4, \pi, 5 \pi / 4,3 \pi / 2,7 \pi / 4)$. In vivo SSFP images of the knee were acquired on the same 3T scanner using a 15-channel knee coil. The imaging parameters were as follows: $192 \times 192 \times 192-\mathrm{mm}^{3}$ field-of-view, $1 \times 1 \times 1-\mathrm{mm}^{3}$ spatial resolution, $\alpha=30^{\circ}, 5.0-\mathrm{ms} \mathrm{TR}, 2.5-\mathrm{ms}$ TE, superior/inferior and anterior/posterior phase-encode dimensions, and eight phase-cycled acquisitions. All protocols were approved by the Ethics Committee of Bilkent University, and written informed consent was obtained from all subjects.

For each region, variable-density sampling patterns were generated in the phase-encode dimensions to yield an acceleration factor of $N$. In vivo datasets were retrospectively undersampled using both common and disjoint sampling strategies. The following acquisitions were used to reconstruct the final images: $\Delta \phi=(0, \pi)$ for $N=2, \Delta \phi=(0, \pi / 2, \pi, 3 \pi / 2)$ for $N=$ $4, \Delta \phi=(0, \pi / 2,3 \pi / 4, \pi, 5 \pi / 4,7 \pi / 4)$ for $N=6$, and $\Delta \phi=$ $(0, \pi / 4 \pi / 2,3 \pi / 4, \pi, 5 \pi / 4,3 \pi / 2,7 \pi / 4)$ for $N=8$. Undersampled data were density compensated, and then reconstructed using compressed sensing. For brain data, the Daubechies-4 wavelet transform, $\lambda_{1}=0.002, \lambda_{2}=0.003$, and 60 iterations were used. For knee data, an identity transform, $\lambda_{1}=0.0005$,
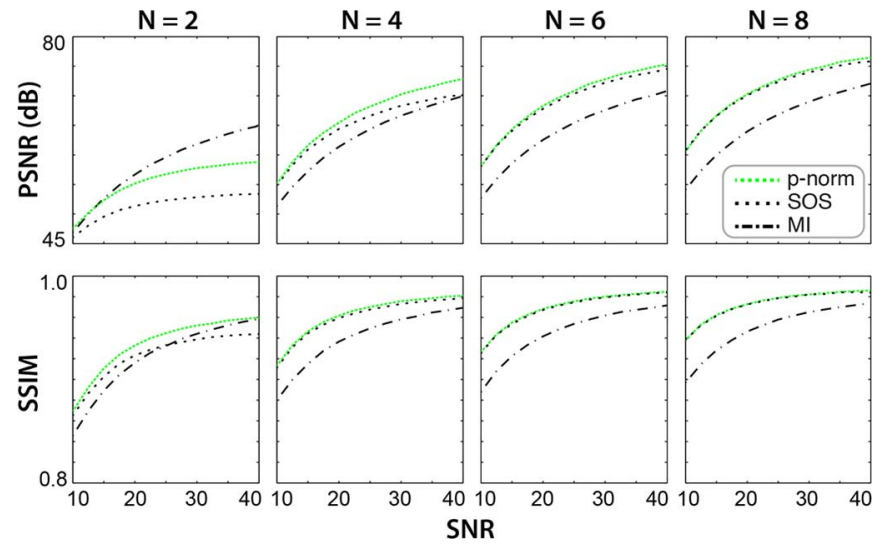

Fig. 2. Total of $N$ phase-cycled and fully-sampled SSFP images $\left(\alpha=30^{\circ}\right.$, $\mathrm{TR} / \mathrm{TE}=5.0 / 2.5 \mathrm{~ms}$ ) were combined using MI, SOS, and p-norm methods. SNR of the maximally bright tissue (CSF) in phase-cycled images was varied from 10 to 40 . The combined image was compared against a reference image (simulated without off-resonant shifts). Two quality metrics were calculated: peak SNR (PSNR, top row) and structural similarity index (SSIM, bottom row). Calculations were repeated for axial, coronal and sagittal brain images, and the resulting metrics were averaged. Quality of the combined image is enhanced for larger $N$ and higher SNR values. In addition, the p-norm method outperforms the MI and SOS methods for $N>2$ at all SNR values.

$\lambda_{2}=0.004$, and 30 iterations were used. Finally, the reconstructed images from separate phase-cycled acquisitions were combined using the p-norm method $(p=4)$.

\section{RESULTS}

\section{A. Simulations}

To evaluate the proposed technique, SSFP images of a digital brain phantom were simulated. Individual and combined phase-cycled images are displayed in Fig. 1. A banding-free SSFP image was also simulated in the absence of off-resonant frequencies. The difference between the combinations and the banding-free image are also shown in Fig. 1(b). As expected, increasing $N$ reduces errors due to residual banding artifacts.

To assess the combination methods, brain phantom images for $N=2,4,6$, and 8 were combined using the MI, SOS, and p-norm methods. The level of artifact suppression (measured with PSNR and SSIM) for separate methods are shown in Fig. 2. PSNR and SSIM for all methods improve for larger $N$ and higher SNR. Furthermore, the p-norm method $(p=4)$ yields higher PSNR and SSIM than the other methods for $N>2$, and comparable performance for $N=2$. These results were valid for all TR/TE, $\alpha$, and $\mathrm{T}_{1} / \mathrm{T}_{2}$ ratios tested here. Thus, the $\mathrm{p}$-norm method was used for all combinations hereafter.

Next, the brain phantom images were undersampled by a factor of $N$ using uniform- and variable-density patterns (see Section II). In common sampling, a single pattern was used across all phase-cycled images. In disjoint sampling, a unique pattern was assigned to each phase-cycled image. Sample combinations of axial and coronal brain images are displayed in Fig. 3. The disjoint sampling strategy yields visibly reduced artifacts compared to common sampling.

To quantitatively assess the image quality for each sampling strategy, two separate analyses were performed. First, the level 


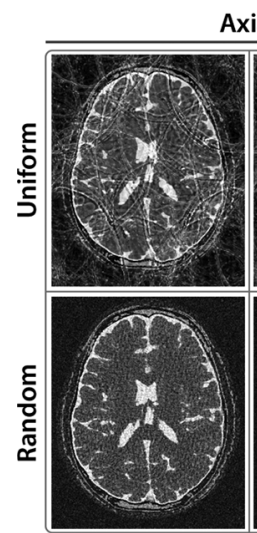

Common
Axial
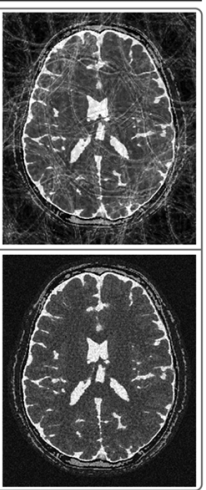

Disjoint

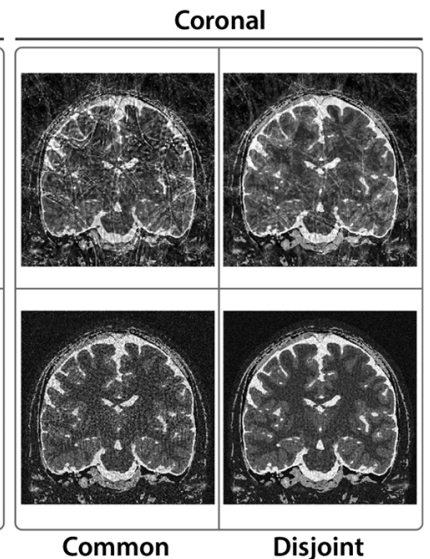

Common

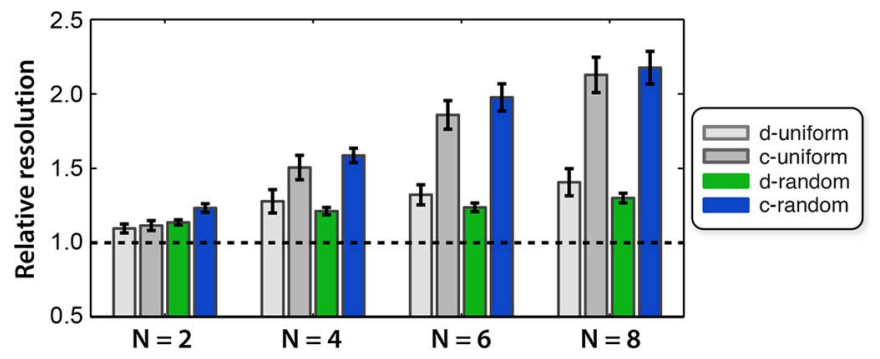

Fig. 5. Effects of four separate sampling strategies on image resolution were assessed. For both uniform- and variable-density random sampling, the patterns were either common (c-uniform, c-random) or disjoint (d-uniform, d-random) across phase-cycles. Relative resolution of each sampling strategy was calculated from the respective line-spread functions (see Section II). Error bars indicate s.e. across spatial orientations. As expected, relative resolution is degraded for higher acceleration factors $(N)$. Disjoint sampling achieves improved resolution compared to common sampling for all $N$, and for both uniform and random patterns.
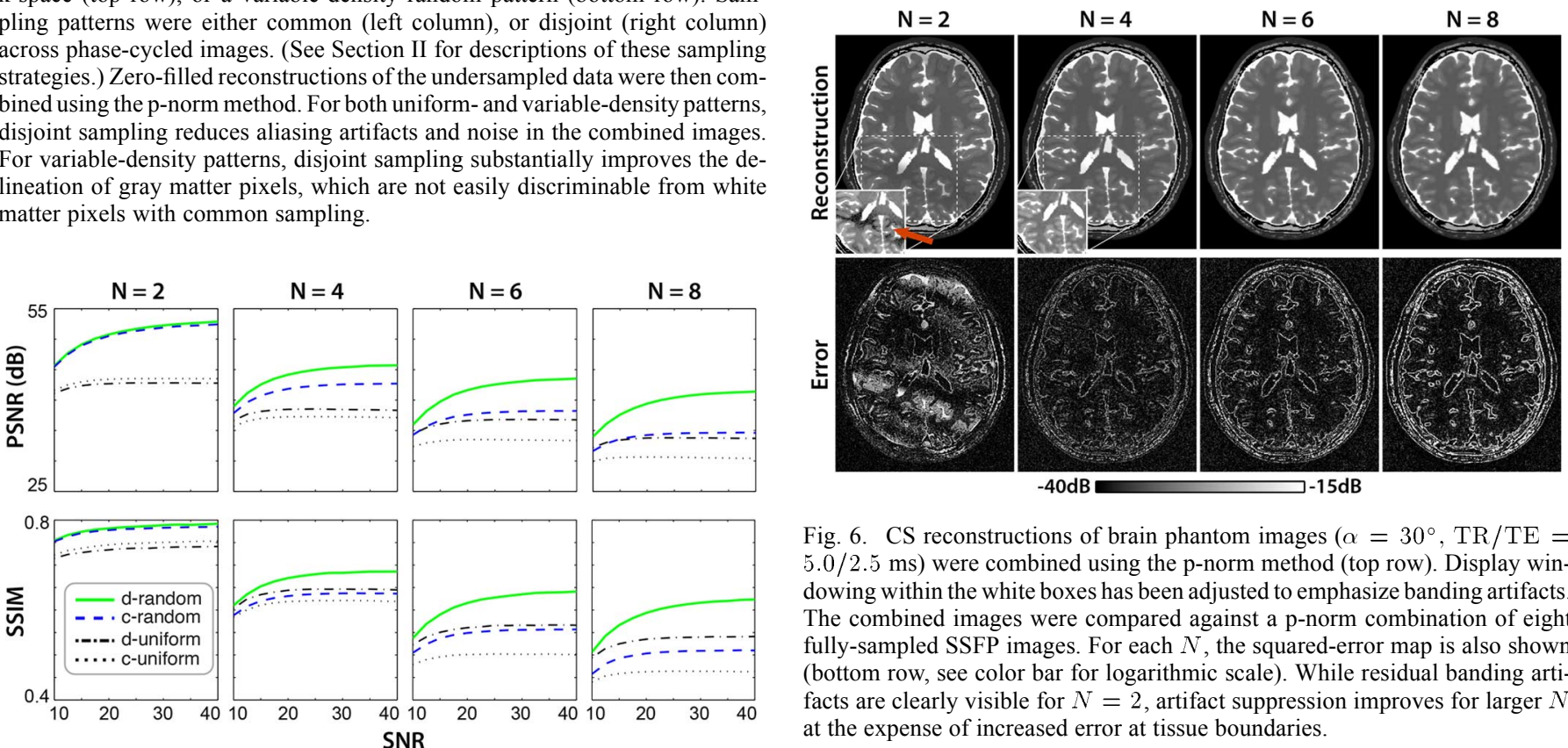

Fig. 4. Total of $N$ phase-cycled brain phantom images $\left(\alpha=30^{\circ}, \mathrm{TR} / \mathrm{TE}=\right.$ $5.0 / 2.5 \mathrm{~ms}$ ) were undersampled by a factor of $N$. For both uniform- and variable-density random sampling, the patterns were either common (c-uniform, c-random) or disjoint (d-uniform, d-random) across phase-cycles. Zero-filled reconstructions of undersampled data were combined using the p-norm method, and compared to the combination of fully-sampled images. Peak SNR (top row) and SSIM (bottom row) indices were calculated for axial, coronal and sagitta brain images, and the resulting metrics were averaged. As expected, the quality of the combined image is reduced larger $N$ due to increased undersampling. The d-random strategy outperforms all other methods in terms of PSNR and SSIM In addition, disjoint sampling across phase-cycles yields higher image quality than common sampling.

of artifacts were evaluated by comparing the combination of undersampled images against a combination of fully-sampled images (Fig. 4). For all $N$, variable-density patterns yield higher PSNR and SSIM than uniform-density patterns. In addition, disjoint sampling across phase-cycled images significantly increases PSNR and SSIM for both types of patterns. These results indicate that lowest level of aliasing artifacts are attained by variable-density patterns with disjoint sampling across phasecycled acquisitions.
Fig. 6. $\mathrm{CS}$ reconstructions of brain phantom images $\left(\alpha=30^{\circ} . \mathrm{TR} / \mathrm{TE}=\right.$ $5.0 / 2.5 \mathrm{~ms}$ ) were combined using the p-norm method (top row). Display windowing within the white boxes has been adjusted to emphasize banding artifacts. The combined images were compared against a p-norm combination of eight fully-sampled SSFP images. For each $N$, the squared-error map is also shown (bottom row, see color bar for logarithmic scale). While residual banding artifacts are clearly visible for $N=2$, artifact suppression improves for larger $N$ at the expense of increased error at tissue boundaries.

Second, the relative resolution for each strategy was measured using high-contrast slanted-edge images (see Section II). Resolution measurements for each strategy are shown in Fig. 5. For both uniform- and variable-density patterns, disjoint sampling significantly enhances spatial resolution for all $N$ compared to common sampling.

We finally assessed the effect of sampling strategies on compressed-sensing reconstructions. The combined reconstructions were compared against a reference combination of fully-sampled brain phantom images $(N=8)$. The reconstructions, and the squared error between the reconstructed and reference images are shown in Fig. 6. Banding artifacts, which are clearly visible for $N=2$, are effectively suppressed for larger $N$. However, larger $N$ also leads to loss of fine image features due to the high degree of undersampling. This result implies that there is an optimal value of $N$ that achieves a favorable trade-off between artifact suppression and image resolution. This prediction is also supported by the PSNR and SSIM measurements between the combined reconstructions and the reference image, 
TABLE II

QuALITY OF CS RECONSTRUCTIONS: Simulated DATA

Results for: $\mathbf{T R}=\mathbf{5} \mathrm{ms}, \alpha=\mathbf{3 0}{ }^{\circ}$, fixed $\mathbf{T}_{1} / \mathbf{T}_{2}$

\begin{tabular}{l|l|cccc}
\hline \multicolumn{2}{c|}{ Number of Acq. } & N = 2 & $\mathbf{N}=\mathbf{4}$ & $\mathbf{N}=\mathbf{6}$ & $\mathbf{N}=\mathbf{8}$ \\
\hline \hline \multirow{2}{*}{ Disjoint } & PSNR (dB) & 59.26 & 65.07 & 58.20 & 51.85 \\
& SSIM (\%) & 93.77 & 94.48 & 93.16 & 91.34 \\
\hline \multirow{2}{*}{ Common } & PSNR & 59.38 & 64.27 & 57.09 & 51.67 \\
& SSIM & 93.81 & 94.31 & 92.30 & 90.00 \\
\hline
\end{tabular}

Results averaged over: $T R=(5,10,15) \mathrm{ms}$, $\alpha=\left(30^{\circ}, 60^{\circ}, 90^{\circ}\right), \pm 20 \%$ deviation in $\mathrm{T}_{1} / \mathrm{T}_{2}$

\begin{tabular}{l|l|llll}
\hline \multicolumn{2}{c|}{ Number of Acq. } & N = 2 & N = 4 & N = 6 & N = 8 \\
\hline \hline \multirow{2}{*}{ Disjoint } & PSNR (dB) & 57.75 & 60.26 & 58.02 & 55.17 \\
& SSIM (\%) & 89.53 & 90.45 & 89.98 & 89.06 \\
\hline \multirow{2}{*}{ Common } & PSNR & 57.78 & 60.02 & 57.31 & 54.61 \\
& SSIM & 89.63 & 90.31 & 89.21 & 87.96 \\
\hline
\end{tabular}

PSNR and SSIM indices measured on CS reconstructions of brain images simulated using a range of pulse sequence and tissue parameters. Indices are reported for a range of total number of acquisitions $(\mathrm{N})$, and common or disjoint sampling strategies.

listed in Table II. Disjoint sampling improves PSNR by $0.37 \pm$ $0.08 \mathrm{~dB}$ (mean \pm s.e. across all tested imaging and relaxation parameters) and SSIM by $0.48 \pm 0.12 \%$ over common sampling. Importantly, PSNR and SSIM values are highest for $N=4$, indicating maximized image quality.

\section{B. In Vivo Experiments}

Our simulation results suggest that disjoint sampling combined with variable-density patterns yields the maximal image quality. To test this prediction in vivo, the proposed technique was demonstrated on SSFP images of the brain and the knee (see Section II). In both regions, compressed-sensing reconstructions were compared against a reference combination of fully-sampled images $(N=8)$. Representative combined images for $N=2,4,6$, and 8 are shown in Fig. 7 along with the squared error between the combinations and the reference image. Visible banding artifacts at $N=2$ are gradually suppressed for larger $N$. At the same time, higher undersampling factors for larger $N$ (particularly $N=8$ ) cause errors around tissue boundaries due to loss of high-spatial-frequency information.

In vivo PSNR and SSIM measurements for both disjoint and common sampling are listed in Table III. Disjoint sampling yields $1.13 \pm 0.57 \mathrm{~dB}($ mean \pm s.e. across $N)$ higher PSNR than common sampling for brain images, and 0.95 $\pm 0.49 \mathrm{~dB}$ higher PSNR for knee images. Disjoint sampling improves SSIM $1.11 \pm 0.55 \%$ (mean \pm s.e.) consistently across $N$ only for knee images. Highest PSNR values for both brain and knee images are obtained using disjoint sampling and $N=4$ (matching with the simulation results). Meanwhile, SSIM values for $N=2$ and $N=4$ are comparable to each other, and higher than SSIM values for larger $N$. This result implies that $N=4$ maintains a near-optimal compromise between suppression of banding artifacts and preservation of detailed image structure.
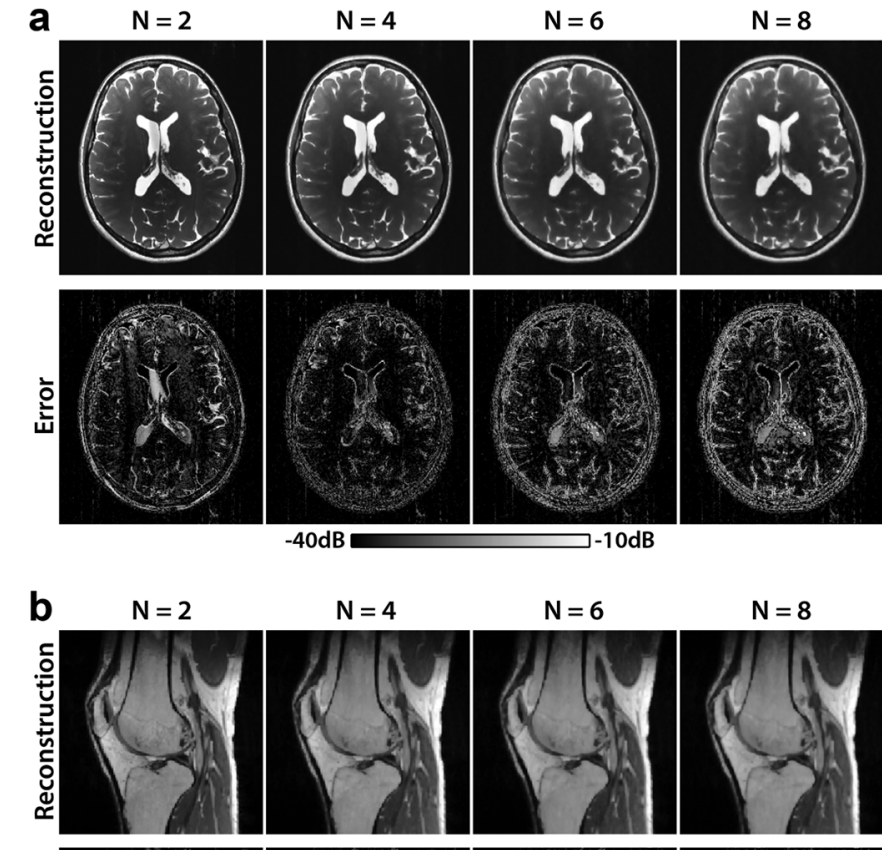

$-40 \mathrm{~dB} \longrightarrow-10 \mathrm{~dB}$
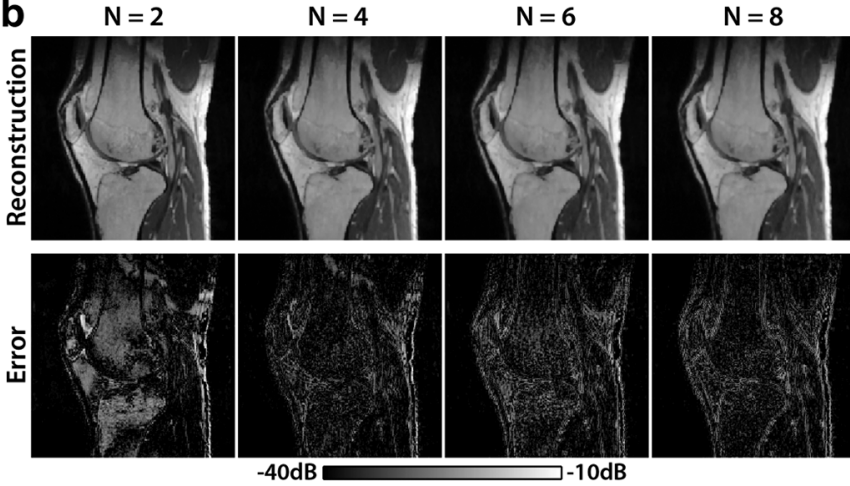

Fig. 7. CS reconstructions of in vivo brain (a) and knee (b) images were combined using the p-norm method (top row). The combined images were compared against a p-norm combination of eight fully-sampled images. For each $N$, the squared-error map is shown in logarithmic scale (bottom row, see color bar). The error is dominated by residual banding artifacts for $N=2$, and by loss of high-spatial-frequency information for $N=8$.

TABLE III

QUALITY OF CS RECONSTRUCTIONS: IN VIVO DATA

\begin{tabular}{l|l|cccc}
\multicolumn{7}{c}{ Brain Data } \\
\hline \multicolumn{2}{c}{ Number of Acq. } & $\mathbf{N}=\mathbf{2}$ & $\mathbf{N}=\mathbf{4}$ & $\mathbf{N}=\mathbf{6}$ & $\mathbf{N}=\mathbf{8}$ \\
\hline \hline \multirow{2}{*}{ Disjoint } & PSNR (dB) & 59.36 & 65.73 & 60.87 & 57.21 \\
& SSIM (\%) & 94.10 & 94.37 & 91.18 & 90.81 \\
\hline \multirow{2}{*}{ Common } & PSNR & 59.40 & 64.97 & 59.23 & 55.01 \\
& SSIM & 93.91 & 93.74 & 92.10 & 89.11 \\
\hline
\end{tabular}

Knee Data

\begin{tabular}{l|l|cccc}
\hline \multicolumn{2}{c|}{ Number of Acq. } & $\mathbf{N}=\mathbf{2}$ & $\mathbf{N}=\mathbf{4}$ & $\mathbf{N}=\mathbf{6}$ & $\mathbf{N}=\mathbf{8}$ \\
\hline \hline \multirow{2}{*}{ Disjoint } & PSNR (dB) & 61.70 & 68.05 & 66.33 & 67.64 \\
& SSIM (\%) & 95.18 & 94.65 & 93.65 & 93.51 \\
\hline \multirow{2}{*}{ Common } & PSNR & 61.65 & 67.43 & 65.26 & 65.59 \\
& SSIM & 95.16 & 93.98 & 92.16 & 91.28 \\
\hline
\end{tabular}

PSNR and SSIM indices measured on CS reconstructions of in vivo brain and knee images. Indices are reported for a range of total number of acquisitions $(\mathrm{N})$, and common or disjoint sampling strategies.

\section{DISCUSSION}

Our simulation and in vivo results indicate that disjoint sampling across phase-cycles improves the quality of combined SSFP images. Note that disjoint sampling patterns are partly nonoverlapping. Thus, the number of unique k-space samples 
TABLE IV

RECONSTRUCTION TIMES

\begin{tabular}{l|cccc}
\hline \multicolumn{1}{c|}{ Number of Acq. } & $\mathbf{N}=\mathbf{2}$ & $\mathbf{N}=\mathbf{4}$ & $\mathbf{N}=\mathbf{6}$ & $\mathbf{N = 8}$ \\
\hline \hline Phantom $(434 \times 434$ matrix $)$ & $24.27 \mathrm{~s}$ & $34.91 \mathrm{~s}$ & $49.18 \mathrm{~s}$ & $64.40 \mathrm{~s}$ \\
\hline Brain data $(218 \times 218)$ & $5.58 \mathrm{~s}$ & $7.37 \mathrm{~s}$ & $10.15 \mathrm{~s}$ & $13.89 \mathrm{~s}$ \\
\hline Knee data $(192 \times 192)$ & $2.26 \mathrm{~s}$ & $2.89 \mathrm{~s}$ & $4.33 \mathrm{~s}$ & $5.93 \mathrm{~s}$ \\
\hline
\end{tabular}

Total reconstruction times are listed for a single slice of simulated phantom and in vivo data. Reconstructions were performed in MATLAB (Mathworks, MA), on a workstation equipped with a $3.4 \mathrm{GHz}$ quad-core Intel E3 processor (Intel Corporation, CA) and $32 \mathrm{~GB}$ of memory.

collected across $N$ phase-cycled acquisitions is greater for disjoint sampling than for common sampling. For the patterns generated here, the collective $\mathrm{k}$-space span for disjoint sampling is $74.1 \%$ at $N=2,65.5 \%$ at $N=4,61.4 \%$ at $N=6$, and $58.0 \%$ at $N=8$. In contrast, the span for common sampling is merely $50.0 \%$ at $N=2,25.0 \%$ at $N=4,16.7 \%$ at $N=6$, and $12.5 \%$ at $N=8$. This fundamental difference helps explain the superior performance of the disjoint sampling strategy.

The proposed technique is primarily demonstrated for brain and knee imaging. However, it could significantly accelerate many other SSFP applications with prominent banding artifacts, such as peripheral angiography [6] and coronary artery imaging [3], [4]. Note that the sampling strategies outlined here are generally applicable to scenarios where multiple acquisitions are combined. For instance, the proposed technique could minimize artifacts for accelerated fat/water separation [44], [45], flow-sensitive angiography [46], and positive-contrast cell tracking [12].

The phantom and in vivo experiments reported here converge on a single optimum of $N=4$ that maximizes image quality. Furthermore, our simulations clearly indicate that this optimal sampling strategy is robust against reasonable variations in repetition time $(5.0-15.0 \mathrm{~ms})$, flip angle $\left(30^{\circ}-90^{\circ}\right)$, SNR $(10-40)$, and tissue parameters $\left( \pm 20 \%\right.$ deviation in $\left.\mathrm{T}_{1} / \mathrm{T}_{2}\right)$. Note, however, that optimum $N$ inevitably depends on several factors including measurement SNR levels, total acquisition time, prescribed image resolution, and reconstruction performance. With higher measurement SNR, increased acquisition time or larger voxels, higher degrees of acceleration may be used without significant loss in image information. Similarly, the advance of improved reconstruction methods that reduce residual errors may shift optimal $N$ to larger values.

Although the proposed technique maintains the scan time of a single acquisition, data pertaining to different phase-cycling increments are collected apart in time. If the overall scan time is longer than several minutes, artifacts due to patient motion could arise. The motion sensitivity could be potentially reduced by interleaving separate acquisitions, and using catalyzation pulses to dampen transient oscillations while switching between phase-cycling increments [15].

The proposed technique reconstructs phase-cycled acquisitions separately prior to the final combination. However, these acquisitions contain shared image structure, and they should be jointly compressible [47], [48]. Thus, reconstructions that enforce the joint sparsity of phase-cycled images could improve image quality for high acceleration factors. Furthermore, the information provided by separate elements in coil arrays could be utilized in conjunction with image sparsity to enable further acceleration factors [47], [49]. Finally, it is possible to shorten TR and improve suppression of banding artifacts by employing partial Fourier acquisitions in the readout dimension in conjunction with the proposed technique [29].

\section{CONCLUSION}

In this work, we proposed an imaging technique to reduce banding artifacts without any increase in scan time compared to a single SSFP acquisition. The technique randomly undersamples acquisitions using unique variable-density patterns for each phase cycle. High quality reconstructions are obtained via compressed sensing, and they are combined using the p-norm method to suppress banding artifacts. Our technique enables rapid and robust SSFP imaging in the presence of magnetic field inhomogeneities.

\section{APPENDIX}

The magnetization terms $M_{o}(r), A(r), B(r)$ in the SSFP signal equation (1) are given as [31]

$$
\begin{aligned}
M_{o}(r) & =\frac{i M_{o} e^{-\frac{T E}{T_{2}(r)}}\left(1-E_{1}(r)\right) \sin \alpha}{1-E_{1}(r) \cos \alpha-\left(E_{1}(r)-\cos \alpha\right) E_{2}^{2}(r)} \\
A(r) & =E_{2}(r) \\
B(r) & =\frac{E_{2}(r)\left(1-E_{1}(r)\right)(1+\cos \alpha)}{1-E_{1}(r) \cos \alpha-\left(E_{1}(r)-\cos \alpha\right) E_{2}^{2}(r)} .
\end{aligned}
$$

Here, $r$ denotes spatial position, TE denotes the echo time, TR denotes the repetition time, and $\alpha$ is the radio-frequency flip angle. $E_{1,2}(r)=e^{-(T R) /\left(T_{1,2}(r)\right)}$, where $T_{1,2}(r)$ denote longitudinal and transverse relaxation times, respectively.

\section{ACKNOWLEDGMENT}

The author would like to thank M. Lustig for sharing his reconstruction routines, and E. U. Saritas for comments and suggestions on the manuscript.

\section{REFERENCES}

[1] H. Y. Carr, "Steady-state free precession in nuclear magnetic resonance," Phys. Rev., vol. 112, pp. 1693-1701, 1958.

[2] D. C. Peters, D. B. Ennis, and E. R. McVeigh, "High-resolution MRI of cardiac function with projection reconstruction and steady-state free precession," Magn. Reson. Med., vol. 48, no. 1, pp. 82-88, 2002.

[3] B. A. Jung, J. Hennig, and K. Scheffler, "Single-breathhold 3D-true FISP cine cardiac imaging," Magn. Reson. Med., vol. 48, no. 5, pp. 921-925, 2002.

[4] K. S. Nayak, B. A. Hargreaves, B. S. Hu, D. G. Nishimura, J. M. Pauly, and C. H. Meyer, "Spiral balanced SSFP cardiac imaging," Magn. Reson. Med., vol. 53, no. 1, pp. 1468-1473, 2005.

[5] J. H. Brittain, E. W. Olcott, A. Szuba, G. E. Gold, G. A. Wright, P. Irarrazaval, and D. G. Nishimura, "Three-dimensional flow-independent peripheral angiography," Magn. Reson. Med., vol. 38, no. 3, pp. 343-354, 1997

[6] T. Cukur, J. H. Lee, N. K. Bangerter, B. A. Hargreaves, and D. G. Nishimura, "Non-contrast-enhanced flow-independent peripheral MR angiography with balanced SSFP," Magn. Reson. Med., vol. 61, no. 6, pp. $1533-1539,2009$

[7] B. A. Hargreaves, G. E. Gold, C. F. Beaulieu, S. S. Vasanawala, D. G. Nishimura, and J. M. Pauly, "Comparison of new methods for magnetic resonance imaging of articular cartilage," Magn. Reson. Med., vol. 49, no. 4, pp. 700-709, 2003. 
[8] S. S. Vasanawala, B. A. Hargreaves, J. M. Pauly, D. G. Nishimura, C. F. Beaulieu, and G. E. Gold, "Rapid musculoskeletal MRI with phasesensitive steady-state free precession: Comparison with routine knee MRI," AJR. Am. J. Roentgenol., vol. 184, pp. 1450-1455, 2004.

[9] O. Bieri, S. Patil, H. H. Quick, and K. Scheffler, "Morphing steadystate free precession," Magn. Reson. Med., vol. 58, pp. 1242-1248, 2007.

[10] I. Koktzoglou, D. Li, and R. Dharmakumar, "Dephased FLAPS for improved visualization of susceptibility-shifted passive devices for realtime interventional MRI," Phys. Med. Biol., vol. 52, no. 1, pp. 277-286, 2007.

[11] C. Heyn, C. V. Bowen, B. K. Rutt, and P. J. Foster, "Detection threshold of single SPIO-labeled cells with FIESTA," Magn. Reson. Med., vol. 53, pp. 312-320, 2005.

[12] T. Çukur, M. Yamada, W. R. Overall, P. Yang, and D. G. Nishimura, "Positive contrast with alternating repetition time SSFP (PARTS): A fast imaging technique for SPIO-labeled cells.," Magn Reson Med., vol. 63, no. 2, pp. 427-437, Feb. 2010.

[13] K. L. Miller, B. A. Hargreaves, J. Lee, D. Ress, R. C. de Charms, and J. M. Pauly, "Functional brain imaging using a blood oxygenation sensitive steady-state," Magn. Reson. Med., vol. 50, no. 4, pp. 675-683, 2003.

[14] K. Scheffler, E. Seifritz, D. Bilecen, R. Venkatesan, J. Hennig, M. Deimling, and E. M. Haacke, "Detection of BOLD changes by means of a frequency-sensitive true FISP technique: Preliminary results," NMR Biomed., vol. 14, no. 7-8, pp. 490-496, 2001.

[15] K. Scheffler, O. Heid, and J. Hennig, "Magnetization preparation during the steady-state: Fat-saturated 3D true FISP," Magn. Reson. Med., vol. 45, no. 6, pp. 1075-1080, 2001.

[16] K. Scheffler and S. Lehnhardt, "Principles and applications of balanced SSFP techniques," Eur. Radiol., vol. 13, no. 11, pp. 2409-2418, 2003.

[17] Y. Zur, M. L. Wood, and L. J. Neuringer, "Motion-insensitive, steadystate free precession imaging," Magn. Reson. Med., vol. 16, no. 3, pp. 444-459, 1990

[18] E. M. Haacke, P. A. Wielopolski, J. A. Tkach, and M. T. Modic, "Steady-state free precession imaging in the presence of motion: Application for improved visualization of the cerebrospinal fluid," Radiology, vol. 175, pp. 545-552, 1990.

[19] N. K. Bangerter, B. A. Hargreaves, S. S. Vasanawala, J. M. Pauly, G. E. Gold, and D. G. Nishimura, "Analysis of multiple-acquisition SSFP," Magn. Reson. Med., vol. 51, no. 5, pp. 1038-1047, 2004.

[20] A. M. Elliott, M. A. Bernstein, H. A. Ward, J. Lane, and R. J. Witte, "Nonlinear averaging reconstruction method for phase-cycle SSFP," Magn. Reson. Imag., vol. 25, pp. 359-364, 2007.

[21] T. Çukur, N. K. Bangerter, and D. G. Nishimura, "Enhanced spectral shaping in steady-state free precession imaging," Magn. Reson. Med., vol. 58, pp. 1216-1223, 2007.

[22] T. Çukur, M. Lustig, and D. G. Nishimura, "Multiple-profile homogenous image combination: Application to phase-cycled SSFP and multicoil imaging," Magn. Reson. Med., vol. 60, pp. 732-738, 2008.

[23] B. Quist, B. A. Hargreaves, T. Çukur, G. R. Morrell, G. E. Gold, and N. K. Bangerter, "Simultaneous fat suppression and band reduction with large-angle multiple-acquisition balanced steady-state free precession.," Magn. Reson. Med., vol. 67, no. 4, pp. 1004-1012, Apr. 2012.

[24] B. A. Hargreaves, "Partially dephased SSFP for elimination of dark bands," in Proceedings of the 16th Annual Meeting of ISMRM, Toronto, 2008, p. 1357.

[25] K. S. Nayak, H.-L. Lee, B. A. Hargreaves, and B. S. Hu, "Wideband SSFP: Alternating repetition time balanced steady state free precession with increased band spacing," Magn. Reson. Med., vol. 58, no. 1, pp. 931-938, 2007.

[26] J. Lee, M. Lustig, D. H. Kim, and J. M. Pauly, "Improved shim method based on the minimization of the maximum off-resonance frequency for balanced steady-state free precession (bSSFP).," Magn. Reson. Med., vol. 61, no. 6, pp. 1500-1506, Jun. 2009.

[27] S. S. Vasanawala, J. M. Pauly, and D. G. Nishimura, "Linear combination steady-state free precession MRI," Magn. Reson. Med., vol. 43, no. 1, pp. 82-90, 2000.
[28] K. T. Block, M. Uecker, and J. Frahm, “Undersampled radial MRI with multiple coils: Iterative image reconstruction using a total variation constraint," Magn. Reson. Med., vol. 57, pp. 1086-1098, 2007.

[29] M. Lustig, D. Donoho, and J. M. Pauly, "Sparse MRI: The application of compressed sensing for rapid MR imaging," Magn. Reson. Med., vol. 58, no. 6, pp. 1182-1195, 2007.

[30] T. Çukur, M. Lustig, and D. G. Nishimura, "Improving non-contrastenhanced steady-state free precession angiography with compressed sensing," Magn. Reson. Med., vol. 61, no. 5, pp. 1122-1131, 2009.

[31] M. L. Lauzon and R. Frayne, "Analytical characterization of RF phasecycled balanced steady-state free precession," Concepts Magn. Reson., vol. 34A, no. 3, pp. 133-143, 2009.

[32] H. W. Korin, S. J. Riederer, A. E. H. Bampton, and R. L. Ehman, "Altered phase encoding order for reduced sensitivity to motion corruption in 3DFT MR imaging," J. Magn. Reson. Imag., vol. 2, no. 1, pp. 687-693, 1992.

[33] F. A. Breuer, M. Blaimer, M. F. Mueller, N. Seiberlich, R. M. Heidemann, M. A. Griswold, and P. M. Jakob, "Controlled aliasing in volumetric parallel imaging (2D CAIPIRINHA).," Magn. Reson. Med., vol. 55, no. 3, pp. 549-556, Mar. 2006.

[34] T. Çukur, M. Lustig, E. U. Saritas, and D. G. Nishimura, "Signal compensation and compressed sensing for magnetization-prepared MR angiography," IEEE Trans. Med. Imag., vol. 30, no. 5, pp. 1017 -1027, May 2011.

[35] D. L. Donoho, M. Elad, and V. N. Temlyakov, "Stable recovery of sparse overcomplete representations in the presence of noise," IEEE Trans. Inf. Theory., vol. 52, no. 1, pp. 6-18, Jan. 2006.

[36] R. C. Gonzalez and R. E. Woods, Digital Image Processing. New York: Addison-Wesley, 1998.

[37] Z. Wang, A. C. Bovik, H. R. Sheikh, and E. P. Simoncelli, "Image quality assessment: From error visibility to structural similarity," IEEE Trans Image Process., vol. 13, no. 4, pp. 600-612, Apr. 2004.

[38] P. D. Burns, "Slanted-Edge MTF for digital camera and scanner analysis," in Proc. PICS Conf., IS\&T, 2000, pp. 135-138.

[39] J. G. Pipe, "Reconstructing MR images from undersampled data: Dataweighting considerations," Magn. Reson. Med., vol. 43, pp. 867-875, 2000 .

[40] B. Aubert-Broche, M. Griffin, G. B. Pike, A. C. Evans, and D. L. Collins, "Twenty new digital brain phantoms for creation of validation image data bases.," IEEE Trans. Med. Imag., vol. 25, no. 11, pp. 1410-1416, Nov. 2006.

[41] J. P. Wansapura, S. K. Holland, R. S. Dunn, and W. S. Ball, "NMR relaxation times in the human brain at 3.0 tesla.," J. Magn. Reson. Imag., vol. 9, no. 4, pp. 531-538, Apr. 1999.

[42] G. E. Gold, E. Han, J. Stainsby, G. Wright, J. Brittain, and C. Beaulieu, "Musculoskeletal MRI at 3.0 T: Relaxation times and image contrast.," AJR Am. J. Roentgenol., vol. 183, no. 2, pp. 343-351, Aug. 2004.

[43] I. Daubechies, "Orthonormal bases of compactly supported wavelets," Comm. Pure Appl. Math., vol. 41, no. 7, pp. 909-996, Oct. 1988.

[44] T. Çukur and D. G. Nishimura, "Fat-water separation with alternating repetition time balanced SSFP," Magn. Reson. Med., vol. 60, pp. 479-484, 2008.

[45] B. A. Hargreaves, N. K. Bangerter, S. S. Vasanawala, A. Shimakawa, J. H. Brittain, and D. G. Nishimura, "Dual-acquisition phase-sensitive SSFP water-fat separation," Magn. Reson. Imag., vol. 24, no. 2, pp. 113-122, 2006.

[46] M. Miyazaki and V. S. Lee, "Nonenhanced MR angiography," Radiology, vol. 248, pp. 20-43, 2008.

[47] R. Otazo, D. Kim, L. Axel, and D. K. Sodickson, "Combination of compressed sensing and parallel imaging for highly accelerated firstpass cardiac perfusion MRI.," Magn. Reson. Med., vol. 64, no. 3, pp. 767-776, Sept. 2010.

[48] M. Akçakaya, P. Hu, M. L. Chuang, T. H. Hauser, L. H. Ngo, W. J. Manning, V. Tarokh, and R. Nezafat, "Accelerated noncontrast-enhanced pulmonary vein MRA with distributed compressed sensing.," J. Magn. Reson. Imag., vol. 33, no. 5, pp. 1248-1255, May 2011.

[49] D. Liang, B. Liu, J. Wang, and L. Ying, "Accelerating SENSE using compressed sensing," Magn. Reson. Med., vol. 62, no. 6, pp. 1574-1584, 2009. 